\title{
A new nonclassicality measure for the quantum states of a bosonic field
}

\author{
Stephan De Bièvre ${ }^{1, *}$, Dmitri Horoshko ${ }^{2,3}$, Giuseppe Patera ${ }^{2}$, and Mikhail Kolobov ${ }^{2}$ \\ ${ }^{1}$ Univ. Lille, CNRS, UMR 8524 - Laboratoire Paul Painlevé, F-59000 Lille, France \\ ${ }^{2}$ Univ. Lille, CNRS, UMR 8523 - PhLAM - Physique des Lasers Atomes et Molécules, F-59000 Lille, \\ France \\ ${ }^{3}$ B. I. Stepanov Institute of Physics, NASB, Nezavisimosti Ave. 68, Minsk 220072 Belarus
}

\begin{abstract}
We review a recently proposed measure of the nonclassicality of a bosonic field, based on the sensitivity of its quasi-probability distributions to ordering of the creation and annihilation operators. We illustrate the new measure by several concrete examples and show its advantages compared to other measures of nonclassicality such as the Wigner function negativity and the entanglement potential.
\end{abstract}

\section{Introduction}

It is well known that quantum theory admits states of the electromagnetic field, not admitted by classical electrodynamics, e.g. the single-photon state and the squeezed state of an optical mode. Thus, all possible states of one optical mode are divided in two classes: states having a classical analog (classical states), and states having no classical analog (nonclassical states). There is a sharp border between these two classes [1]: for a classical state the Glauber-Sudarshan $P$-function satisfies the requirements for a probability density, in particular, positive-definiteness. Negativity of the $P$-function indicates that the corresponding state is non-classical. However, determination of the $P$-function at all points of the complex plain is a difficult task, when this function is reconstructed from the experimental data. Moreover, the $P$-function of a nonclassical state may be highly singular. It is strongly desirable to have a parameter which (i) indicates that the measured state is non-classical, and (ii) says how strongly nonclassical the state is, i.e. how far this state is from the class of classical states. A parameter satisfying the first requirement is known as a "nonclassicality witness", while the second requirement corresponds to a "nonclassicality measure". Various nonclassicality measures have been proposed in the last decades, however some of them may be regarded as nonclassicality witnesses only, since the amount of nonclassicality which they ascribe to a quantum state does not always correspond to the amount of resources that is necessary for creation of this nonclassical state. In this work we review a recently proposed measure of nonclassicality [2] and compare it with several other such measures on the class of highly nonclassical Schrödinger cat states of an optical field.

\footnotetext{
*e-mail: Stephan.De-Bievre@univ-lille.fr
} 


\section{The s-ordered entropy}

The quantum state of a single mode optical field is fully characterized by its density operator $\rho$ having a one-to-one correspondence to the $s$-ordered quasiprobability distributions [3]:

$$
W_{s}(\alpha)=\frac{1}{\pi^{2}} \int \chi_{s}(\xi) e^{\xi^{*} \alpha-\xi \alpha^{*}} d^{2} \xi
$$

where $s$ is a real parameter and the $s$-ordered characteristic function $\chi_{s}(\xi)$ is defined as

$$
\chi_{s}(\xi)=\operatorname{Tr}\left\{\rho e^{\xi a^{\dagger}-\xi^{*} a}\right\} e^{s|\xi|^{2} / 2},
$$

with $a$ being the photon annihilation operator for the mode under consideration. Note that our normalisation of $W_{s}(\alpha)$ is different from that of Cahill and Glauber [3] by the additional factor $\frac{1}{\pi}$, resulting in

$$
\int W_{s}(\alpha) d^{2} \alpha=\chi_{s}(0)=\operatorname{Tr} \rho=1
$$

as expected for a quasiprobability distribution.

Different values of the ordering parameter $s$ correspond to different orderings of the operators $a$ and $a^{\dagger}$, when the average of a function $f\left(a, a^{\dagger}\right)$ is replaced by the average of $f_{s}\left(\alpha, \alpha^{*}\right)$, calculated with a quasiprobability. In particular, $s=1$ correponds to the normal ordering and the Glauber-Sudarshan $P$-function, $s=0$ correponds to the symmetric ordering and the Wigner function, and $s=-1$ correponds to the anti-normal ordering and the Husimi $Q$-function.

The spread in phase space of a quasiprobability distribution can be characterized by its entropy. We select from different entropic measures the second-order Renyi enropy, and define the $s$-ordered entropy of a quantum state as

$$
H(s)=-\ln \left\{\pi \int W_{s}^{2}(\alpha) d^{2} \alpha\right\},
$$

where the integration is performed over the full complex plane. Substituting Eq. (1) into Eq. (4) we obtain an alternative definition for the $s$-ordered entropy

$$
H(s)=-\ln \left\{\frac{1}{\pi} \int\left|\chi_{s}(\xi)\right|^{2} d^{2} \xi\right\} .
$$

The normalisation of the entropy in Eq. (4) is chosen so that

$$
H(0)=-\ln \left\{\pi \int W_{0}^{2}(\alpha) d^{2} \alpha\right\}=-\ln \mathcal{P}(\rho),
$$

where $\mathcal{P}(\rho)$ is the purity of a quantum state defined as $\mathcal{P}(\rho)=\operatorname{Tr} \rho^{2}$. Thus, for a pure state the $s$-ordered entropy always passes through the origin: $H(0)=0$, while for a mixed state it always passes above the origin: $H(0)>0$.

The $s$-ordered entropy is invariant with respect to translation in the phase space. Indeed, for two density operators related by $\tilde{\rho}=D(\alpha) \rho D^{\dagger}(\alpha)$, where $D(\alpha)=\exp \left(\alpha a^{\dagger}-\alpha^{*} a\right)$, the characteristic functions are related by $\tilde{\chi}_{s}(\xi)=\chi_{s}(\xi) \exp \left(2 i \operatorname{Im}\left\{\xi \alpha^{*}\right\}\right)$, and the entropy Eq. (5) is not affected by this transformation, since it depends only on the modulus of the characteristic function.

The $s$-ordered entropy has simple analytic expressions for basic quantum states of one optical mode. For a coherent state $\left|\alpha_{0}\right\rangle$ it is equal to

$$
H_{\mathrm{coh}}(s)=\ln (1-s)
$$


independently of $\alpha_{0}$. For a Fock state $|n\rangle$ it is given by

$$
H_{\text {Fock }}(s)=-\ln \left\{\int_{0}^{\infty} L_{n}^{2}(x) e^{(s-1) x} d x\right\}=-\ln \left[\frac{(1+s)^{n}}{(1-s)^{n+1}} P_{n}\left(\frac{1+s^{2}}{1-s^{2}}\right)\right],
$$

where $L_{n}(x)$ is the Laguerre polynomial and $P_{n}(x)$ is the Legendre polynomial. For a squeezed vacuum state with the squeezing parameter $r$ [4] it is given by

$$
H_{\mathrm{sq}}(s)=\frac{1}{2} \ln \left(e^{2 r}-s\right)\left(e^{-2 r}-s\right) \text {. }
$$

For a thermal state with $\langle n\rangle$ photons on average

$$
H_{\text {therm }}(s)=\ln (2\langle n\rangle+1-s) .
$$

These dependencies are shown in Fig. 1.

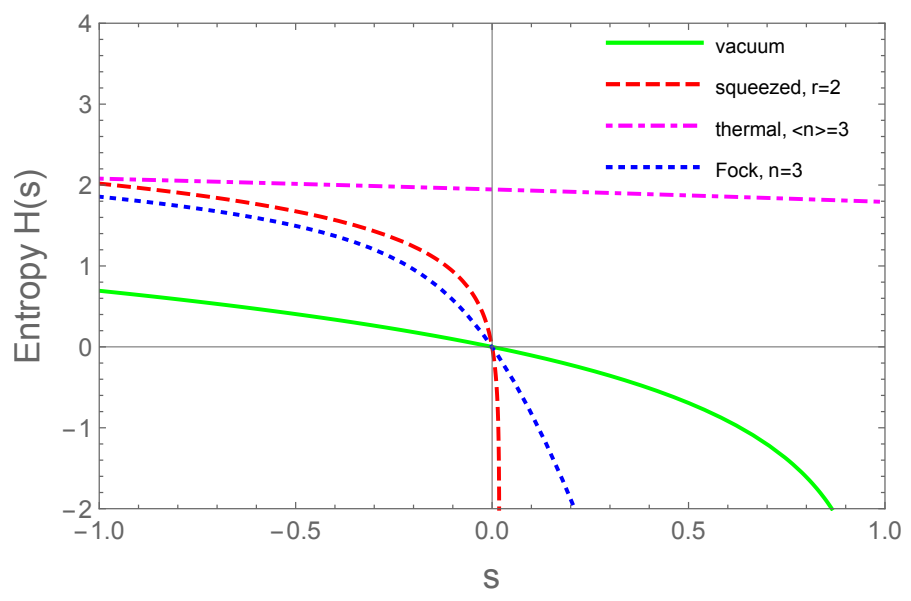

Figure 1. Renyi entropy $H(s)$ of the quasiprobability distribution as function of the ordering parameter $s$. Entropy of pure state passes through the origin, since $H(0)=-\ln \mathcal{P}(\rho)$. Different states exhibit quite different sensitivities to the ordering parameter.

Analytic expressions can be also obtained for the states which are known as Schrödinger cat states of the single-mode field. For an even (odd) coherent state $\left|\alpha_{ \pm}\right\rangle \propto|\alpha\rangle \pm|-\alpha\rangle$ [5, 6], it is given by

$$
H_{\text {even/odd }}(s)=-\ln \left\{\frac{1}{1-s}\left[1+\frac{e^{-\frac{4|\alpha|^{2}}{1-s}}-e^{-4|\alpha|^{2}}+e^{\frac{4 s|\alpha|^{2}}{1-s}}-1}{2\left(1 \pm e^{-2|\alpha|^{2}}\right)^{2}}\right]\right\} .
$$

For a rotationally-invariant circular state (RICS), which represents a multi-component Schrödinger cat $[6,7]$

$$
\left|\mathbf{c}_{q}\right\rangle=\frac{1}{N \sqrt{\tilde{g}(q)}} \sum_{m=0}^{N-1} e^{i 2 \pi m q / N}\left|\alpha_{0} e^{-i 2 \pi m / N}\right\rangle
$$

where

$$
\tilde{g}(k)=\frac{1}{N} \sum_{m=0}^{N-1} e^{-i 2 \pi k m / N} \exp \left\{\left|\alpha_{0}\right|^{2}\left(e^{i 2 \pi m / N}-1\right)\right\}
$$


this entropy can be expressed in finite sums:

$$
H_{\mathrm{RICS}}(s)=-\ln \left\{\frac{1}{1-s} \frac{e^{-2\left|\alpha_{0}\right|^{2}}}{N^{4} \tilde{g}(q)^{2}} \sum_{m, n, l, k=0}^{N-1} e^{-i 2 \pi(m-n-l+k) q / N} e^{-\frac{s}{1-s} \alpha_{m}^{*} \alpha_{n}-\frac{s}{1-s} \alpha_{k}^{*} \alpha_{l}+\frac{1}{1-s} \alpha_{m}^{*} \alpha_{l}+\frac{1}{1-s} \alpha_{k}^{*} \alpha_{n}}\right\} .
$$

Note that the even and the odd coherent states are particular cases of RICS with $N=2$ and $q=0$ and $q=1$ respectively.

Let us establish some general properties of the considered entropy. It is well known [3] that in the range $s \in[-1,0]$ the $s$-ordered quasiprobability distribution, defined by Eq. (1) is a bounded square-integrable function on the full complex plane. Besides, in the same range it satisfies the relation

$$
\int W_{s}^{2}(\alpha) d^{2} \alpha \leq \frac{1}{\pi}
$$

wherefrom it follows that the $s$-ordered entropy, defined by Eq. (4), is non-negative in the same range of parameter values.

It can be shown that in the range $s \in[-1,0]$ the minimal value of the $s$-ordered entropy over all possible states is that of a coherent state, Eq. (7). Indeed, let us consider two modes $A$ and $B$ in identical states $\rho_{A}=\rho_{B}$. Now for the characteristic function of mode $A$ we can write

$$
\begin{aligned}
\left|\chi_{0}(\xi)\right|^{2} & =\operatorname{Tr}_{A}\left\{\rho_{A} e^{\xi a^{\dagger}-\xi^{*} a}\right\} \operatorname{Tr}_{B}\left\{\rho_{B} e^{-\xi b^{\dagger}+\xi^{*} b}\right\}=\operatorname{Tr}_{A B}\left\{\rho_{A} \rho_{B} e^{\xi\left(a^{\dagger}-b^{\dagger}\right)-\xi^{*}(a-b)}\right\} \\
& =\operatorname{Tr}_{A B}\left\{\rho_{A} \rho_{B} B_{A B}^{\dagger} e^{\xi \sqrt{2} b^{\dagger}-\xi^{\dagger} \sqrt{2} b} B_{A B}\right\}=\tilde{\chi}_{0}(\sqrt{2} \xi),
\end{aligned}
$$

where $a$ and $b$ are the photon annihilation operators for the modes $A$ and $B$ respectively, $B_{A B}=$ $e^{\frac{\pi}{4}\left(a^{\dagger} b-b^{\dagger} a\right)}$ is the beam-splitter operator for these two modes, and $\tilde{\chi}_{0}(\xi)$ is the characteristic function for the state

$$
\tilde{\rho}_{B}=\operatorname{Tr}_{A}\left\{B_{A B} \rho_{A} \rho_{B} B_{A B}^{\dagger}\right\} .
$$

Now, substituting Eq. (17) into Eq. (5), we obatin

$$
H(s)=-\ln \left\{\frac{1}{\pi} \int \tilde{\chi}_{0}(\sqrt{2} \xi) e^{s|\xi|^{2}} d^{2} \xi\right\}=-\ln \left\{\frac{\pi}{2} \tilde{W}_{s}(0)\right\},
$$

where $\tilde{W}_{s}(0)$ is the $s$-ordered quasiprobability distribution for the state Eq. (17). It is known [3] that for $s \leq 0$ the absolute value of the $s$-ordered quasiprobability is less than or equal to $\frac{2}{\pi}(1-s)^{-1}$. It follows, that on the same range holds

$$
H(s) \geq \ln (1-s) .
$$

We note, that physically the state Eq. (17) is the result of a destructive interference of the quantum field in the state $\rho_{A}$ with itself. For a coherent state it is always the vacuum.

\section{The ordering sensitivity}

The Husimi distribution is generally wider than the Wigner one for generic quantum states, and therefore is characterized by a higher entropy. More generally, $H(s)$ is non-increasing in $s \in[-1,0]$, because its derivative is non-positive in this range, which can be obtained by differentiating Eq. (5) by $s$. In a similar way we prove that it is concave.

The rate with which the entropy grows when the parameter $s$ goes from 0 to -1 can be characterised by the first derivative at the origin. Thus, we define the "ordering sensitivity" of a quantum state as [2]

$$
S_{\mathrm{o}}=-H^{\prime}(0)
$$


It is easy to obtain from Eq. (7) that for a coherent state (and in particular for the vacuum state), $S_{\mathrm{o}}^{\text {coh }}=1$, which can be used as unit of sensitivity. From Eqs. (8)-(10) we obtain the sensitivies of the corresponding states:

$$
S_{\mathrm{o}}^{\text {Fock }}=2 n+1, \quad S_{\mathrm{o}}^{\mathrm{sq}}=\cosh 2 r, \quad S_{\mathrm{o}}^{\text {thermal }}=\frac{1}{2\langle n\rangle+1},
$$

wherefrom it follows that the sensitivities of the Fock and the squeezed states (except for the trivial case of vacuum) are higher than 1 and grow with growing energy. On the contrary, the sensitivity of the thermal state (except for the same trivial case) is less than 1 and decreases to 0 with growing energy, which means that for a thermal state with high temperature the noncommutativity of the operators $a$ and $a^{\dagger}$ can be neglected. Such a behaviour allows one to suggest that the ordering sensitivity shows the importance of the operator non-commutativity and may therefore be a good measure of the quantum state nonclassicality.

Indeed, it has been recently proven [2] that for any quantum state having a classical analog, i.e. a state whose $P$-function can be considered as a probability distribution [1], the ordering sensitivity is always less than or equal to 1 . Moreover, a geometric interpretation of the ordering sensitivity has been established [2]. Let us define the inner product in the operator space of single-mode optical field for two operators $A$ and $B$ as

$$
\langle A, B\rangle=\frac{1}{2} \operatorname{Tr}\left(\left[A^{\dagger}, Q\right][Q, B]+\left[A^{\dagger}, P\right][P, B]\right),
$$

where $Q=\frac{1}{\sqrt{2}}\left(a^{\dagger}+a\right)$ and $P=\frac{i}{\sqrt{2}}\left(a^{\dagger}-a\right)$. It has been shown that $\|A\| \|=\langle A, A\rangle^{\frac{1}{2}}$ is a well-defined operator norm. Now the operator ordering sensitivity of state $\rho$ can be written as $S_{\mathrm{o}}=\|\tilde{\rho}\| \|^{2}$, where $\tilde{\rho}=\rho / \sqrt{\operatorname{Tr}\left(\rho^{2}\right)}$. It means that for any classical state $\rho$ its renormalized density operator $\tilde{\rho}$ lies inside the unit ball $\|\tilde{\rho}\| \| \leq 1$. We denote the set of all classical states as $C$ and define the distance from $\rho$ to $C$ by $d(\rho, C)=\inf _{\sigma \in C}\|\tilde{\rho}-\tilde{\sigma}\| \|$. The nonclassicality of $\rho$ is defined as

$$
\mathcal{N}(\rho)=d(\rho, C)
$$

It has been shown [2] that for the norm defined above

$$
\|\tilde{\rho} \mid\|-1 \leq \mathcal{N}(\rho) \leq\|\tilde{\rho}\| \|,
$$

hence, if $\|\tilde{\rho}\| \mid \gg 1$, then $\|\tilde{\rho}\| \mid$ provides a very good estimate of $\mathcal{N}(\rho)$. Thus, the condition $S_{\text {o }}>1$ is a sufficient criterion of nonclassicality and with growing $S_{\mathrm{o}}$ it approaches the distance-based measure of nonclassicality, defined by Eq. (23).

For pure states the following relation holds [2]:

$$
S_{\mathrm{o}}=\left\langle(Q-\langle Q\rangle)^{2}\right\rangle+\left\langle(P-\langle P\rangle)^{2}\right\rangle=2\left\langle\left(a^{\dagger}-\left\langle a^{\dagger}\right\rangle\right)(a-\langle a\rangle)\right\rangle+1,
$$

that is, the ordering sensitivity is given by the energy of quantum fluctuations. From this formula we find easily the ordering sensitivity for various states belonging to the family of Schrödinger cat states. For the even and odd coherent states [5] which are generated in the microwave [6] and the optical domains [8]

$$
S_{\mathrm{o}}^{\text {even/odd }}=2|\alpha|^{2} \frac{1-e^{-4|\alpha|^{2}}}{\left(1 \pm e^{-2|\alpha|^{2}}\right)^{2}}+1
$$

and for the Yurke-Stoler state $|\alpha\rangle+i|-\alpha\rangle$, [9-11], which can be generated by self-modulation in a Kerr medium, demonstrated recently in the microwave domain [12],

$$
S_{\mathrm{o}}^{\mathrm{YS}}=2|\alpha|^{2}\left(1-e^{-4|\alpha|^{2}}\right)+1
$$


For any coherent amplitude $\alpha \neq 0$ these states are nonclassical and their nonclassicality grows quadratically in $|\alpha|$ with the growth of the field amplitude.

Ordering sensitivity of a RICS, which is a multi-component Schrödinger cat [6, 7], is shown in Fig. 2 together with other two measures of nonclassicality, the Wigner function negativity [13] and the entanglement potential [14].

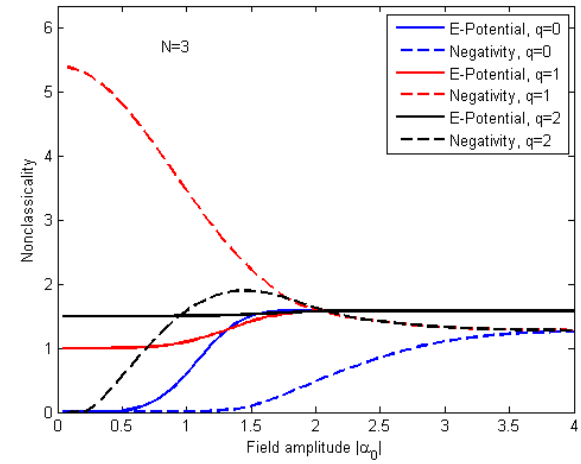

(a)

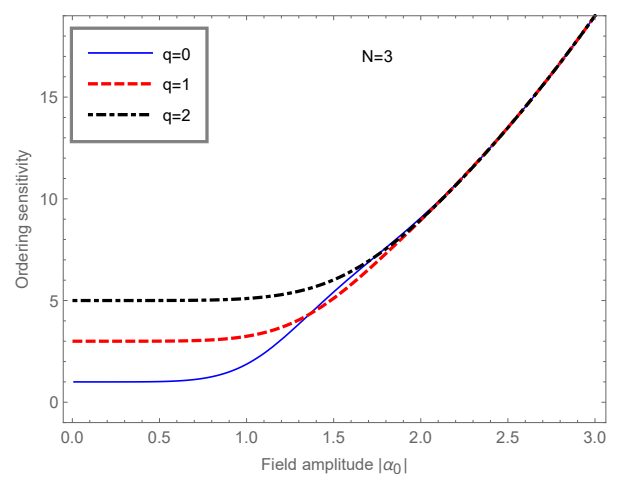

(b)

Figure 2. Nonclassicality of a superposition of 3 coherent states (RICS) for various values of the relative phase, determined by $q$. (a) Entanglement potential (solid lines) and Wigner function negativity (dashed lines). (b) Ordering sensitivity. Both the entanglement potential and the negativity are saturated at sufficiently high values of the cat size $\left|\alpha_{0}\right|$. In contrast, the ordering sensitivity grows continuously with the growth of the cat size, as expected for a nonclassicality measure.

Figure 2 shows the principal difference between the ordering sensitivity and the other two measures of nonclassicality. While the entanglement potential and the negativity are saturated at certain $\left|\alpha_{0}\right|$ and do not change any more with the growth of the cat size, the ordering sensitivity predicts that a growing Schrödinger cat becomes more and more nonclassical, as intuitively expected. It should be noted that some recently proposed measures of "quantum macroscopicity" $[15,16]$ possess the same property, they are growing with the cat size. Comparison of our approach with these measures will be done elsewhere.

\section{Conclusions}

We have reviewed the recently proposed measure of nonclassicality based on the sensitivity of the quasi-probability distribution to to ordering of the creation and annihilation operators of a bosonic field [2]. Comparison of the new measure with such measures as the Wigner function negativity and the entanglement potential on the class of Schrödinger cat states demonstrated the clear advantage of the new measure - its growth with the growth of the cat size.

\section{Acknowledgments}

This work was supported in part by the Labex CEMPI (ANR-11-LABX-0007-01) and by the Nord-Pas de Calais Regional Council and FEDER through the Contrat de Projets ÉtatRégion (CPER), and in part by the European Union's Horizon 2020 research and innovation programme under grant agreement No 665148 (QCUMbER). 


\section{References}

[1] U.M. Titulaer, R.J. Glauber, Phys. Rev. 140, B676 (1965)

[2] S.D. Bièvre, D.B. Horoshko, G. Patera, M.I. Kolobov, arXiv: 1809.02047

[3] K.E. Cahill, R.J. Glauber, Phys. Rev. 177, 1882 (1969)

[4] M.I. Kolobov, Rev. Mod. Phys. 71, 1539 (1999)

[5] V. Dodonov, I. Malkin, V. Man'ko, Physica 72, 597 (1974)

[6] S. Haroche, J.M. Raimond, Exploring the Quantum: Atoms, Cavities and Photons (Oxford University Press, 2006)

[7] D.B. Horoshko, S. De Bièvre, M.I. Kolobov, G. Patera, Phys. Rev. A 93, 062323 (2016)

[8] A. Ourjoumtsev, F. Ferreyrol, R. Tualle-Brouri, P. Grangier, Nature Physics 5, 189 (2009)

[9] B. Yurke, D. Stoler, Phys. Rev. Lett. 57, 13 (1986)

[10] D.B. Horoshko, S.Y. Kilin, Opt. Express 2, 347 (1998)

[11] D.B. Horoshko, S.Y. Kilin, J. Exp. Theor. Phys. 90, 733 (2000)

[12] G. Kirchmair, B. Vlastakis, Z. Leghtas, S.E. Nigg, H. Paik, E. Ginossar, M. Mirrahimi, L. Frunzio, S.M. Girvin, R.J. Schoelkopf, Nature 495, 205 (2013)

[13] A. Kenfack, K. Życzkowski, J. Opt. B 6, 396 (2004)

[14] J.K. Asbóth, J. Calsamiglia, H. Ritsch, Phys. Rev. Lett. 94, 173602 (2005)

[15] C.W. Lee, H. Jeong, Phys. Rev. Lett. 106, 220401 (2011)

[16] B. Yadin, V. Vedral, Phys. Rev. A 93, 022122 (2016) 